\title{
Uso de leitos hospitalares privados por sistemas públicos de saúde na resposta à Covid-19
}

\author{
Use of private hospital beds by public health systems in response to \\ Covid-19
}

Adriano Massuda', Renato Tasca², Ana Maria Malik1

DOI: 10.1590/0103-11042020E416

RESUMO Neste estudo, examinaram-se estratégias adotadas por países com sistemas públicos de saúde que expandiram a oferta de leitos por meio da utilização de hospitais privados na resposta à Covid-19. Utilizou-se estudo de casos selecionados para explorar o contexto institucional em que as medidas foram implementadas, os instrumentos de gestão utilizados e como se caracterizou a ação governamental em oito países: Austrália, Espanha, Irlanda, Itália, Chile, México e Peru, além do Brasil. Esta análise pode auxiliar a identificar mecanismos de gestão de sistema de saúde necessários para a coordenação de ações governamentais para resposta a situações de Emergência em Saúde Pública (ESP), bem como para aperfeiçoar a governança dos sistemas de saúde na relação entre os setores público e privado.

PALAVRAS-CHAVE Administração de serviços hospitalares. Administração de saúde pública. Serviços médicos de emergência. Parcerias Público-Privadas.

\begin{abstract}
In this study, strategies adopted by countries with public health systems that expanded the supply of beds through the use of private hospitals in response to Covid-19 were examined. The study of selected cases was used to explore the institutional context in which measures were implemented, the management tools used and how to characterize government action in eight countries: Australia, Spain, Ireland, Italy, Chile, Mexico and Peru, in addition to Brazil. This analysis can help to identify health system management mechanisms necessary for the coordination of government actions to respond to Public Health Emergency situations, as well as improve the governance of health systems in the relationship between public and private sectors.
\end{abstract}

KEYWORDS Hospital facilities administration. Public health administration. Emergency medical services. Public-Private Sector Partnerships.

\footnotetext{
1 Fundação Getulio Vargas (FGV), Escola de Administração de Empresas de São Paulo (Eaesp) - São Paulo (SP), Brasil.

a.massuda@gmail.com

2 Organização Mundial da Saúde (OMS), Organização Pan-Americana da Saúde (Opas) - Brasília (DF), Brasil.
} 


\section{Introdução}

Emergências em Saúde Pública (ESP) testam a resiliência de sistemas de saúde e exigem de governos capacidade para implementar ações rápidas e eficientes dirigidas a mitigar impactos em saúde e oferecer assistência e orientação à população, ${ }^{\mathbf{1} 2}$. Na pandemia provocada pelo novo coronavírus SARS CoV-2 (Covid-19), um dos maiores impactos sobre o sistema de saúde foi o rápido aumento na demanda por internações hospitalares, especialmente em Unidades de Terapia Intensiva (UTI) ${ }^{\mathbf{3}, 4}$. Isso exigiu dos gestores de sistemas de saúde a adoção de diferentes estratégias para expandir a capacidade instalada de oferta assistencial ${ }^{5}$.

Entre as estratégias observadas para reorganizar a rede assistencial, visando a absorver o aumento da demanda hospitalar, destacaram-se: 'mudança de rotina', com adiamento de procedimentos, cirurgias e internações eletivas; 'mudança de perfil', por meio da redefinição da vocação de hospitais ou alocação de alas hospitalares para destinação de leitos exclusivos para internação de pacientes Covid19; 'ampliação da estrutura assistencial permanente', mediante a transformação de leitos de enfermaria em leitos de maior complexidade, com ênfase em leitos destinados a cuidados críticos; e 'ampliação de estrutura assistencial temporária', por meio do aluguel de hotéis ou montagem de hospitais de campanha para casos de menor complexidade.

Além dessas alternativas, também se verificou que alguns países fizeram uso de leitos em hospitais privados na resposta pública à Covid-196. Essa medida mostrou-se efetiva, pois, em muitas situações, esses leitos já dispunham de estrutura, equipamentos e pessoal para adequado funcionamento, conferindo agilidade às ações e reduzindo a necessidade de investimentos para a construção de novos leitos. Porém, a relação entre os setores público e privado na saúde tem particularidades que variaram de acordo com o contexto de cada país e o modelo de organização de seus respectivos sistemas de saúde, podendo ser geradoras de inequidades no uso dos recursos disponíveis?.

Neste estudo, foram examinadas estratégias adotadas por países com sistemas públicos de saúde que expandiram a oferta de leitos hospitalares por meio da utilização de serviços privados na resposta à pandemia. Buscou-se descrever o contexto institucional em que as medidas foram implementadas, os instrumentos de gestão utilizados e como se caracterizou o modo de ação governamental. Esta análise pode auxiliar a coordenação de ações governamentais em resposta à ESP, bem como oferecer lições para aperfeiçoar a organização e a governança dos setores público e privado nos sistemas de saúde num cenário pós-Covid.

\section{Material e métodos}

Utilizaram-se como método o estudo de casos selecionados e a análise qualitativa dos dados dos países estudados. Buscou-se selecionar uma amostra intencional de países com sistemas públicos de saúde que ampliaram a utilização de hospitais privados na resposta à Covid-19 e que pudessem representar modos distintos de ação governamental no uso de recursos privados pelo poder público. Para identificar os países e coletar dados de pesquisa, realizou-se busca em websites de órgãos governamentais, organismos internacionais e imprensa, complementada por entrevistas com informantes-chave. A busca documental foi realizada entre março e maio de 2020, e foram consultados 14 informantes-chave, entre os quais, pesquisadores e gestores de sistemas de saúde, do Brasil e de países selecionados.

Em seguida, buscou-se caracterizar o contexto institucional, com informações sobre os sistemas de saúde dos países analisados com relação a recursos financeiros alocados na saúde (ano base: 2015): gasto per capita em U\$ PPA, percentual do Produto Interno Bruto (PIB), percentual do gasto público e privado e percentual de desembolso direto sobre gasto privado; e recursos humanos e 
físicos disponíveis: número de médicos e de leitos hospitalares e percentual de leitos públicos (último ano com dados disponíveis). Para coleta desses dados, foram consultadas informações disponíveis nos websites dos escritórios regionais da Organização Mundial da Saúde (OMS), entre os quais: Organização Pan-americana de Saúde (Opas) ${ }^{8}$ e OMS Europa $^{9}$, Organização para a Cooperação e Desenvolvimento Econômico (OCDE) ${ }^{\mathbf{1 0}}$, Perfil Internacional de Sistemas de Saúde ${ }^{11} \mathrm{e}$ Datasus, do Ministério da Saúde ${ }^{\mathbf{1 2}}$.

Por fim, procedeu-se a uma análise qualitativa, buscando caracterizar aspectos da organização do sistema de saúde, com relação a governança, financiamento e participação do setor privado, instrumentos de gestão utilizados para uso de recursos hospitalares privados e o modo de ação governamental em cada um dos países analisados.

\section{Resultados}

Foram encontradas oito experiências de países que expressam diferentes modos de ação governamental no uso de leitos privados por sistemas públicos. Entre eles, um da Oceania: Austrália; três da Europa: Espanha, Irlanda, Itália; e três da América Latina: Chile, México, Peru, além do Brasil.

Na tabela 1, encontram-se dados sobre recursos financeiros e físicos disponíveis nos sistemas de saúde.

Tabela 1. Recursos financeiros e físicos disponíveis no sistema de saúde

\begin{tabular}{|c|c|c|c|c|c|c|c|c|}
\hline & Austrália * & Espanha * & Irlanda * & Itália * & Chile $\star \star$ & 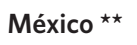 & Peru $\star \star$ & Brasil $\star \star$ \\
\hline População em milhões (2018) & 24,9 & 46,7 & 4,8 & 60,5 & 18,2 & 130,7 & 32,5 & 210,8 \\
\hline $\begin{array}{l}\text { Gasto per capita em saúde U\$ } \\
\text { PPA }\end{array}$ & $4,381.2$ & $3,024.6$ & $4,301.9$ & $3,129.3$ & $1,903.1$ & $1,080.1$ & 671.0 & $1,391.5$ \\
\hline \% Gasto total em relação ao PIB & 9,3 & 8,8 & 7,1 & 8,8 & 8,1 & 5,9 & 5,2 & 8,8 \\
\hline $\begin{array}{l}\text { \% Gasto público em saúde rela- } \\
\text { ção ao PIB }\end{array}$ & 6,4 & 6,2 & 5,3 & 6,5 & 4,9 & 3,1 & 3,2 & 3,8 \\
\hline $\begin{array}{l}\text { \% Gasto público em saúde sobre } \\
\text { total }\end{array}$ & 68,8 & 70,5 & 74,6 & 73,9 & 60,5 & 52,5 & 61,5 & 43,2 \\
\hline $\begin{array}{l}\text { \% Gasto privado em saúde rela- } \\
\text { ção ao PIB }\end{array}$ & 2,9 & 2,6 & 1,8 & 2,3 & 3,2 & 2,8 & 2 & 5 \\
\hline $\begin{array}{l}\text { \% Gasto privado em saúde rela- } \\
\text { ção ao total }\end{array}$ & 31,2 & 29,5 & 25,4 & 26,1 & 39,5 & 47,5 & 38,5 & 56,8 \\
\hline $\begin{array}{l}\text { \% Desembolso direto em saúde } \\
\text { sobre total }\end{array}$ & 18,2 & 24 & 17,7 & 21,2 & 31,0 & 40,8 & 30,9 & 20,3 \\
\hline Número de médicos (mil) & 90,4 & 180,6 & 15,3 & 241,5 & 32,4 & 267,9 & 40,6 & $460,2^{\star \star \star}$ \\
\hline Médicos por 10.000 hab. & 36.1 & 38.7 & 31.8 & 39.9 & 18.0 & 24.0 & 12.7 & 22.0 \\
\hline $\begin{array}{l}\text { Número de leitos hospitalares } \\
\text { (mil) }\end{array}$ & 92,8 & 138,5 & 14,2 & 192,5 & 92,4 & 168,8 & 52,0 & $441,8^{\star \star \star}$ \\
\hline $\begin{array}{l}\text { Número de leitos por } 10.000 \\
\text { habitantes }\end{array}$ & 37 & 30 & 29 & 32 & 51 & 13 & 16 & 21 \\
\hline \% leitos públicos sobre total & $66,5 \%$ & $68,7 \%$ & $85,9 \%$ & $66,7 \%$ & $31,0 \%$ & $73,9 \%$ & $x$ & $70,0 \%$ \\
\hline
\end{tabular}

Fonte: Elaboração própria.

*OCDE - Organização para a Cooperação e Desenvolvimento Econômico.

**Plisa - Plataforma de Información en Salud para las Américas.

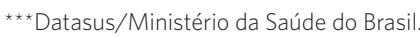


A seguir, será apresentada uma síntese sobre o contexto institucional de cada um dos sistemas de saúde e os instrumentos utilizados para a ampliação do uso de leitos privados na resposta à Covid-19.

\section{Austrália}

O sistema público de saúde australiano é universal, e os três níveis de governo são coletivamente responsáveis pela prestação de assistência à saúde"11. O governo federal financia e apoia os estados na prestação de serviços. Os governos estaduais são responsáveis pela gestão de hospitais públicos, serviços de ambulância, serviços comunitários e de saúde mental"1․ Os governos locais atuam na prestação de programas preventivos, como imunização. Apesar de o financiamento público corresponder a $6,4 \%$ do PIB e representar $68,8 \%$ do gasto total, o setor privado tem importante participação no sistema de saúde, sendo responsável por quase $60 \%$ das cirurgias realizadas do País e por dispor de um terço dos leitos de UTI existentes ${ }^{\mathbf{1 0}}$.

Para enfrentar a pandemia da Covid-19, o governo federal australiano assinou um acordo com representantes dos hospitais privados e das associações médicas e de enfermagem do País, que permitiu colocar 34 mil leitos privados à disposição do sistema público ${ }^{13}$. Esse acordo auxiliou a manter a receita do setor, reduzida pelo cancelamento das cirurgias eletivas. A parceria também mobilizou cerca de 105 mil profissionais de saúde, dos quais, 57 mil são da área de enfermagem ${ }^{14}$. O acordo nacional foi adaptado a cada estado. Em Victoria e Queensland, as parcerias incluíram planejamento conjunto de resposta às necessidades, compras coletivas de equipamentos, medicamentos, insumos e intercâmbio de profissionais de saúde ${ }^{\mathbf{1 5}}$; na Austrália do Sul, restringiram-se à contratação de leitos privados ${ }^{16}$.

\section{Espanha}

O sistema de saúde espanhol é composto de três subsistemas, que coexistem: o Sistema Nacional de Saúde (SNS), complementado por fundos mútuos para os funcionários públicos das forças armadas e do judiciário, e mutualidades focadas na assistência a acidentes e em doenças ocupacionais`. O SNS é universal, financiado por impostos, e a governança é regional, espelhando a divisão administrativa do País em Comunidades Autônomas? O gasto público em saúde é de 6,2\% do PIB, correspondendo a 70,5\% do total em saúde, e a maior parte dos leitos hospitalares é pública ${ }^{11}$. O setor privado é suplementar ao público.

No enfrentamento à Covid-19, o setor privado de saúde teve importante papel no apoio ao SNS, porém, a relação foi variável em cada região. Em geral, o número de centros de saúde e de leitos hospitalares foi ampliado e as unidades reformuladas para abrigar pacientes com a Covid-19. Seguindo o Decreto-Lei que definiu medidas urgentes na esfera econômica e para a proteção da saúde pública ${ }^{17}$, leitos privados de UTI foram estatizados provisoriamente, ficando disponíveis para atender pacientes do sistema público quando este tivesse sua capacidade esgotada ${ }^{18}$.

A ocupação dos leitos privados, entretanto, foi bastante variada, alcançado $100 \%$ dos leitos disponíveis em algumas regiões, enquanto em outras houve baixo ou quase nenhum uso ${ }^{\mathbf{1 9}, 20}$. Além disso, o modo de organização da rede assistencial foi diferenciado. Certas regiões definiram que hospitais públicos seriam totalmente dedicados aos pacientes de Covid, enquanto os privados passaram a cuidar das demais causas de internação, abrindo oportunidades para fortalecer a integração entre os setores num cenário pós-Covid-1921.

\section{Irlanda}

A Irlanda dispõe de um sistema universal de saúde, financiado por impostos e taxas?. Todos os residentes no País têm direito a receber assistência médica prestada no sistema público de saúde. O sistema privado de saúde é suplementar ao público. O País conta com 5,2 leitos de UTI por 100.000 habitantes, a maioria públicos, enquanto a média da comunidade 
europeia é de 11,5 por $100.000^{10}$. O gasto público em saúde é de $5,3 \%$ do PIB, correspondendo a $74,6 \%$ do gasto total ${ }^{10}$.

Na resposta à pandemia da Covid-19, todos os cerca de 2.000 leitos hospitalares (14\% do total), nove laboratórios e centenas de profissionais de saúde do setor privado foram requisitados para atuar no sistema público de saúde 22 . O Estado manteve o controle e a administração das instalações privadas de saúde durante os meses de março e maio, período de maior demanda de casos da pandemia no País ${ }^{23}$. O arranjo foi bem avaliado e poderá ser retomado caso ocorra novo aumento de $\operatorname{casos}^{\mathbf{2 4}}$.

\section{Itália}

O sistema nacional de saúde italiano é universal e organizado regionalmente, sendo papel do governo central definir um pacote nacional de benefícios estatutários a ser oferecido em todas as regiões ${ }^{9,11}$. As 19 regiões do País e duas províncias autônomas têm a responsabilidade de organizar a prestação de serviços de saúde por meio de unidades de saúde locais, com autonomia na forma de fazê-lo. A maior parte dos leitos hospitalares é pública. $O$ gasto público em saúde é de 6,5\% do PIB e corresponde a $73,9 \%$ do gasto total ${ }^{10}$. O setor privado é suplementar ao público.

Para responder a situações de emergência, um decreto presidencial foi publicado contendo medidas urgentes com relação à contenção e ao manejo da emergência epidemiológica da Covid-19. Aplicável em todo o território nacional, o decreto permitiu a requisição, pelo poder público, de estruturas, equipamentos e profissionais de saúde do setor privado ${ }^{25}$, e abriu espaço para diálogo e construção de acordos entre os governos regionais e o setor privado, representado pelas associações locais de hospitais privados ${ }^{26}$.

Em muitos casos, as unidades privadas foram utilizadas para receber pacientes do sistema público com diagnósticos que não fossem de Covid, permitindo que as estruturas públicas se concentrassem no atendimento a pacientes com quadros respiratórios ${ }^{\mathbf{2 7}}$. Em outras regiões, com menor capacidade de internação de pacientes críticos no setor público, unidades privadas foram dedicadas exclusivamente para atendimento de pacientes de Covid ${ }^{28}$. Também houve acordos do tipo 'fila única', que contemplam hospitalização em unidades públicas ou privadas de pacientes com todos os diagnósticos, não apenas Covid 29,30 .

\section{Chile}

O Chile apresenta um sistema de saúde segmentado, que inclui um seguro público (Fundo Nacional de Saúde - Fonasa), um privado (Instituições de Seguridade Social - Isapre), substitutivo ao público, e outros seguros específicos para as Forças Armadas ${ }^{8}$. A prestação de serviços públicos de saúde é realizada por secretarias ministeriais regionais. O gasto público em saúde é de $4,9 \%$ do PIB e corresponde a $60,5 \%$ do total. O setor privado, porém, concentra a maior parte dos leitos hospitalares ${ }^{10}$.

Em 2009, na pandemia do H1N1, o Ministério da Saúde do Chile criou um dispositivo para uso de recursos privados pelo sistema de saúde público chamado de UGCC (Unidad de Gestión Centralizada de Camas), com vistas a coordenar o uso de leitos para pacientes críticos, no setor público e no setor privado ${ }^{31}$. Quando as unidades públicas de saúde chegam à saturação, os leitos do setor privado passam, automaticamente, a ser utilizados. A UGCC ordena o fluxo de pacientes graves seguindo uma classificação de complexidade, dando prioridade para a ocupação dos leitos públicos. Na resposta à Covid-19, o governo chileno publicou um decreto que atribui à UGCC a autoridade de regulação de todos os leitos para pacientes críticos existentes no País ${ }^{32}$. Não se verifica resistência a essa medida por parte do setor privado, e os valores pagos pelas internações são pactuados previamente entre as partes ${ }^{33,34}$. 


\section{México}

O sistema de saúde mexicano é segmentado, composto por instituições de seguridade social do setor público que cobrem trabalhadores do setor formal; o Sistema de Proteção Social em Saúde, que atende a população não coberta pela previdência social e o setor privado, que presta serviços à população com capacidade de pagamento direto ${ }^{8}$. $\mathrm{O}$ gasto público em saúde é de $3,1 \%$ do PIB, pouco superior ao privado - 52,5\% do total. Observa-se alta proporção de desembolso direto para pagamento de despesas com saúde: $40 \%$ do gasto privado ${ }^{10}$. A maior parte dos leitos hospitalares está no setor público.

Em resposta à Covid-19, o governo federal e a Associação Nacional de Hospitais Privados assinaram um acordo de colaboração no qual a rede de hospitais privados disponibiliza $50 \%$ de seus leitos e equipamentos para atender pacientes não Covid-19, cobertos exclusivamente pelos seguros públicos, a fim de liberar leitos no setor público para o atendimento de pacientes com Covid-1935. A estratégia, denominada 'Todos Juntos contra a Covid-19', colocou 3.115 leitos em hospitais privados à disposição para a população geral ${ }^{36}$. O pagamento pelos serviços aí prestados será feito pelo setor público, aos preços acordados para a compra de serviços entre a seguridade social e o departamento de saúde do governo federal ${ }^{37}$. O contrato é temporário e tem validade de um mês.

\section{Peru}

O sistema de saúde do Peru é segmentado, divido em subsetor público, que dirige a maior parte das unidades de saúde públicas do País e presta serviços a populações pobres por meio do sistema de Seguro Saúde Integral; um sistema de seguridade social, que possui a segunda maior rede do País e atende exclusivamente assalariados e familiares; e os serviços de saúde das Forças Armadas e da Polícia Nacional ${ }^{8}$. O gasto público em saúde é de $3,2 \%$ do PIB e corresponde a $61,5 \%$ do total.
O subsetor privado atende principalmente a população de maior renda, sendo $30 \%$ dos gastos privados feitos por desembolso direito ${ }^{8}$.

No Peru, o decreto legislativo estabeleceu o 'intercâmbio de benefícios', permitindo que pacientes sejam tratados em unidades com vagas disponíveis, independentemente se públicas, privadas ou da previdência social, a valores pré-definidos ${ }^{38}$. Graças a essa medida, todas as pessoas sem cobertura privada ou da previdência social têm acesso ao sistema integrado. Isso inclui pessoas em situação irregular, como migrantes. Essa medida está respaldada pelo Decreto de Emergência do Peru, que autoriza o Executivo a impor aos hospitais privados a execução de "serviços extraordinários por sua duração ou natureza"39.

\section{Brasil}

No Brasil, o Sistema Único de Saúde (SUS) é universal e descentralizado, com responsabilidades divididas entre as três esferas de governo. Cabe ao governo federal a definição e o financiamento de políticas nacionais de saúde; aos estados, a organização regional; e aos municípios, a gestão da prestação da maior parte dos serviços. Apesar de o setor privado ser constitucionalmente complementar ao SUS, $56,8 \%$ do gasto em saúde é privado, o que corresponde a $5 \%$ do PIB, dos quais, $20,35 \%$ por desembolso direto. $\mathrm{O}$ gasto público é de $3,8 \%$ do PIB. Apesar de o SUS contar com a da maioria dos leitos hospitalares, a maior parte dos leitos de UTI encontra-se no setor privado.

Em razão da desigualdade no número de leitos de UTI entre os setores público e privado, esteve em debate no País a implementação de uma 'fila única' para acesso aos leitos de UTI para atendimento a pacientes com Covid-19. Isso permitiria a hospitalização de pacientes do SUS em leitos privados, quando a capacidade pública estivesse esgotada ${ }^{40}$. No entanto, associações de planos de saúde, hospitais privados e a Agência Nacional de Saúde Suplementar (ANS) se opuseram ao compartilhamento de leitos ${ }^{41}$. Até o mês de 
agosto de 2020 não houve registro de ação federal para orientar o uso de recursos privados para ampliar a capacidade do SUS.

Por outro lado, verificam-se iniciativas em estados, como Rio Grande do Sul, Espírito Santo e Maranhão, que publicaram, respectivamente, edital de chamamento público para leitos privados atenderem ao SUS, portaria que estabeleceu a possibilidade de uso de recursos privados quando a rede pública for insuficiente e decreto determinando a requisição administrativa dos serviços privados em caso de necessidade ${ }^{\text {2-44 }}$. Em âmbito municipal, a Prefeitura de Curitiba publicou decreto estabelecendo condições e regras para a requisição de bens e serviços de assistência à saúde na pandemia, e a de São Paulo publicou decreto estabelecendo parâmetros para abrir até $20 \%$ da rede privada para atender pacientes do SUS45,46. O quadro 1 apresenta uma síntese dos resultados encontrados.

Quadro 1. Quadro síntese

\begin{tabular}{|c|c|c|c|}
\hline País & Contexto institucional & Instrumentos de gestão & Tipo de ação governamental \\
\hline Austrália & $\begin{array}{l}\text { Sistema nacional sob administração } \\
\text { regional } \\
\text { Financiamento predominantemente } \\
\text { público } \\
\text { Setor privado com forte atuação na } \\
\text { prestação de serviços }\end{array}$ & $\begin{array}{l}\text { Acordo nacional e subacordos re- } \\
\text { gionais para uso de leitos privados } \\
\text { pelo sistema público } \\
\text { Coordenação do acesso aos leitos } \\
\text { definido em cada estado/território }\end{array}$ & $\begin{array}{l}\text { Negociação nacional e acor- } \\
\text { dos regionais }\end{array}$ \\
\hline Espanha & $\begin{array}{l}\text { Sistema nacional sob administração } \\
\text { regional } \\
\text { Financiamento predominantemente } \\
\text { público } \\
\text { Setor privado suplementar }\end{array}$ & $\begin{array}{l}\text { Decreto de emergência no país } \\
\text { aplicável por cada região } \\
\text { Coordenação do acesso aos leitos } \\
\text { definida em cada comunidade } \\
\text { autônoma }\end{array}$ & $\begin{array}{l}\text { Determinação nacional e } \\
\text { implantação regional }\end{array}$ \\
\hline Irlanda & $\begin{array}{l}\text { Sistema nacional de saúde sob adminis- } \\
\text { tração federal } \\
\text { Financiamento predominantemente } \\
\text { público } \\
\text { Setor privado suplementar }\end{array}$ & $\begin{array}{l}\text { Requisição total de serviços pri- } \\
\text { vados } \\
\text { Coordenação nacional do acesso } \\
\text { aos leitos }\end{array}$ & $\begin{array}{l}\text { Determinação nacional e } \\
\text { implantação nacional }\end{array}$ \\
\hline Itália & $\begin{array}{l}\text { Sistema nacional de saúde sob adminis- } \\
\text { tração regional } \\
\text { Financiamento predominantemente } \\
\text { público } \\
\text { Setor privado suplementar }\end{array}$ & $\begin{array}{l}\text { Decreto de emergência no país } \\
\text { permite requisição de serviços do } \\
\text { setor privado } \\
\text { 'Fila única' para hospitalização } \\
\text { instituída por região para todos os } \\
\text { diagnósticos }\end{array}$ & $\begin{array}{l}\text { Determinação nacional e } \\
\text { implantação regional }\end{array}$ \\
\hline Chile & $\begin{array}{l}\text { Sistema misto, que inclui um seguro } \\
\text { público e privado } \\
\text { Financiamento predominantemente } \\
\text { público } \\
\text { Setor privado substitutivo }\end{array}$ & $\begin{array}{l}\text { Decreto presidencial autorizou uso } \\
\text { de leitos privados pelo sistema } \\
\text { público } \\
\text { Regulação do acesso aos leitos por } \\
\text { UGCC (Unidad de Gestión Centra- } \\
\text { lizada de Camas) }\end{array}$ & $\begin{array}{l}\text { Determinação nacional e } \\
\text { implantação nacional }\end{array}$ \\
\hline México & $\begin{array}{l}\text { Sistema segmentado, composto por } \\
\text { seguridade social e sistema de Proteção } \\
\text { Social públicos } \\
\text { Financiamento privado semelhante ao } \\
\text { público } \\
\text { Setor privado substitutivo ao público }\end{array}$ & $\begin{array}{l}\text { Acordo entre o governo federal e a } \\
\text { Associação Nacional de Hospitais } \\
\text { Privados, por meio do qual a rede } \\
\text { de hospitais privados disponibiliza } \\
50 \% \text { de seus leitos para atender } \\
\text { pacientes não Covid-19 }\end{array}$ & $\begin{array}{l}\text { Negociação nacional e im- } \\
\text { plantação nacional }\end{array}$ \\
\hline
\end{tabular}


Quadro 1. (cont.)

\begin{tabular}{|c|c|c|c|}
\hline País & Contexto institucional & Instrumentos de gestão & Tipo de ação governamental \\
\hline Peru & $\begin{array}{l}\text { Sistema segmentado, composto por } \\
\text { seguridade social pública } \\
\text { Financiamento predominantemente } \\
\text { público } \\
\text { Setor privado substitutivo ao público }\end{array}$ & $\begin{array}{l}\text { Decreto legislativo estabeleceu o } \\
\text { "intercâmbio de benefícios" } \\
\text { Pacientes acessam unidades com } \\
\text { vagas disponíveis, independente- } \\
\text { mente se públicas, privadas ou da } \\
\text { previdência social }\end{array}$ & $\begin{array}{l}\text { Determinação nacional e } \\
\text { implantação nacional }\end{array}$ \\
\hline Brasil & $\begin{array}{l}\text { Sistema nacional sob gestão municipal } \\
\text { predominante } \\
\text { Financiamento predominantemente } \\
\text { privado } \\
\text { Setor privado suplementar ao público }\end{array}$ & $\begin{array}{l}\text { Editais, portarias e decretos publi- } \\
\text { cados por municipais e estaduais }\end{array}$ & $\begin{array}{l}\text { Ausência de coordenação } \\
\text { nacional } \\
\text { Atuação localizada por esta- } \\
\text { dos e municípios }\end{array}$ \\
\hline
\end{tabular}

Fonte: Elaboração própria.

\section{Análise e discussão}

As experiências analisadas demonstram que o uso de leitos em hospitais privados por sistemas públicos pode ser uma alternativa para a rápida expansão da capacidade hospitalar na resposta à Covid-19. Entretanto, o modo de utilização é variado, sendo influenciado pelo contexto institucional, pela estrutura do sistema de saúde e, especialmente, pela ação governamental. Entre as experiências analisadas, podem-se observar três tipos de ação governamental.

Um primeiro tipo é ação nacional de 'determinação' da requisição de serviços privados, embasada em leis para situações de emergência ou decreto presidencial. A requisição pode ser total, como na Irlanda; conforme a necessidade apresentada pelo sistema público, como no Chile e no Peru; ou decidida regionalmente, como na Espanha e na Itália. Nessas experiências, a regulação de acesso ao leito é feita por meio de 'fila-única' coordenada pelo sistema público de saúde, nacional ou regionalmente.

Um segundo tipo de ação nacional é a 'negociação' entre o governo federal e representantes do setor privado hospitalar, sendo estabelecidos acordos para colocar um determinado número de leitos privados à disposição para uso do sistema público de saúde. Na experiência da Austrália, o acordo nacional foi adaptado regionalmente, permitindo aprofundar a integração público-privada de acordo com as necessidades de cada região. Nesses casos, ocorreu uma expansão da capacidade hospitalar pública sem necessidade de fila única.

Por fim, um terceiro tipo de ação é realizada por níveis subnacionais de governo, diante da ausência de coordenação nacional, como ocorre na resposta à Covid no Brasil. Nesse caso, a expansão da capacidade hospitalar por meio do uso de serviços privados é limitada ao tipo de ação definido em cada local, que varia desde a contratação de número determinado de leitos até a requisição conforme necessidade.

A presente análise, entretanto, apresenta limitações, que precisam ser exploradas em estudos futuros. Por exemplo, se existe correlação entre orientação política de governos, estrutura de Estado, modelo de financiamento e organização dos sistemas de saúde no modo de ação governamental sobre o uso de recursos privados na resposta à Covid-19. Além disso, é preciso aprofundar a análise sobre algumas questões relativas à relação público-privada em situações de ESP: 
- Dilema ético-institucional: se houver vagas disponíveis em hospitais privados, como negar acesso em meio a uma Pandemia?

- Responsabilidade sanitária do setor privado: esgotada a capacidade pública, a disponibilidade de serviços privados mediante medidas compensatórias adequadas não deveria ser uma responsabilidade social dessas empresas?

- Remuneração: como estabelecer remuneração adequada para os hospitais privados, visando a reembolsar os custos reais?

- Equidade: como garantir que o paciente do sistema público receba cuidado com a mesma qualidade oferecida no setor privado?

\section{Considerações finais}

As experiências estudadas demonstraram que existem diferentes possibilidades de uso de estruturas hospitalares privadas por sistemas públicos de saúde para promover rápida ampliação da capacidade assistencial em situações de ESP. A forma da utilização, porém, é influenciada pelo contexto e pela política institucional estabelecida em cada país e pelos instrumentos de gestão utilizados para operacionalizar a relação público-privada. Sistemas públicos de saúde fortes, com adequado financiamento público, governança e capacidade de alocação de recursos têm maior capacidade de coordenar a relação entre os setores público e privado, ampliando a capacidade assistencial sem comprometer a equidade no acesso a serviços de saúde. As lições apreendidas no enfrentamento da Covid-19 devem ser utilizadas para fortalecer sistemas públicos de saúde num cenário pós-pandemia.

\section{Colaboradores}

Massuda A (0000-0002-3928-136X)*, Tasca R (0000-0002-1407-5914)* e Malik AM (00000002-0813-8886)* contribuíram igualmente para a elaboração do manuscrito. 


\section{Referências}

1. Kruk ME, Myers M, Varpilah ST, et al. What is a resilient health system? Lessons from Ebola. The Lancet. 2015; 385(9980):1910-1912.

2. Organização Mundial da Saúde. Public health emergencies: preparedness and response. Annual report on the implementation of the International Health Regulations [internet]. 2005. [acesso em 2019 abr 4]. Disponível em: https://apps.who.int/ iris/bitstream/handle/10665/328559/A72_8-en. pdf? sequence $=1 \&$ isAllowed $=y$.

3. Legido-Quigley H, Mateos-García JT, Campos VR, et al. The resilience of the Spanish health system against the COVID-19 pandemic. The Lancet. 2020; 5(5):e251-e252.

4. Remuzzi A, Remuzzi G. COVID-19 and Italy: what next? The Lancet. 2020; 395(10231):1225-8.

5. Organização Mundial da Saúde, Organização Pan-Americana da Saúde. Recomendaciones para la Reorganización y Ampliación Progresiva de los Servicios de Salud para la Respuesta a la Pandemia de COVID-19 [internet]. [acesso em 2020 maio 20]. Disponível em: https://www.paho.org/es/documentos/ recomendaciones-para-reorganizacion-ampliacion-progresiva-servicios-salud-para-respuesta.

6. Tasca R, Massuda A. Estratégias para reorganização da Rede de Atenção à Saúde em resposta à Pandemia COVID-19: a experiência do Sistema de Saúde Italiano na região de Lazio. APS em revista. 2020; 2(1):20-7.

7. Santos IS. Evidência sobre o mix público-privado em países com cobertura duplicada: agravamento das iniquidades e da segmentação em sistemas nacionais de saúde. Ciênc. Saúde Colet. 2011; (16):2743-2752.

8. Organização Mundial da Saúde, Organização Pan-Americana da Saúde. PLISA Plataforma de Información en Salud para las Américas [internet]. [acesso em 2020 maio 20]. Disponível em: https://www. paho.org/data/index.php/es/?option=com_content $\&$ view $=$ article $\&$ id=515:indicadoresviz $\&$ Itemid=347.
9. Organização Mundial da Saúde, Escritório Regional para Europa. Países [internet]. [acesso em 2020 maio 20]. Disponível em: http://www.euro.who.int/ en/countries.

10. Organização para a Cooperação e Desenvolvimento Econômico. Country Health Profiles 2019. [acesso em 2020 maio 20]. Disponível em: http://www. oecd.org/health/country-health-profiles-EU.htm.

11. The Commonwealth Fund. International Health Care System Profiles [internet]. [acesso em 2020 maio 20]. Disponível em: https://international.commonwealthfund.org/.

12. Brasil. Ministério da Saúde. DATASUS [internet]. [acesso em 2020 maio 20]. Disponível em: http:// www2.datasus.gov.br/DATASUS/index.php?area=02.

13. Biggs A. COVID-19: Major partnership with private hospitals [internet]. Parliament of Australia. 2020 [acesso em 2020 maio 20]. Disponível em: https:// www.aph.gov.au/About_Parliament/Parliamentary_ Departments/Parliamentary_Library/FlagPost/2020/ April/Agreement_with_private_hospitals.

14. ABC News. Private hospitals to free up thousands of beds in deal with Federal Government to tackle coronavirus [internet]. ABC News. 2020 mar 31. [acesso em 2020 maio 20]. Disponível em: https://www.abc. net.au/news/2020-03-31/coronavirus-agreement-to-keep-private-hospitals-open/12106496.

15. Australian Competition and Consumer Commission. Private and public hospitals to cooperate on COVID-19 in Victoria and Queensland. Accc. 2020 [acesso em 2020 maio 20]; (69):1. Disponível em: https://www.accc.gov.au/media-release/private-and-public-hospitals-to-cooperate-on-covid-19-in-victoria-and-queensland.

16. Government of South Australia. Historic alliance between Public and Private hospitals to defeat COVID-19 [internet]. 2020 [acesso em 2020 maio 20]. Disponível em: https://www.premier.sa.gov.au/news/ 
media-releases/news/historic-alliance-between-public-and-private-hospitals-to-defeat-covid-19.

17. Espanha. Real Decreto 463 de 11 de março de 2020. Real Decreto-ley 6/2020, de 10 de marzo, por el que se adoptan determinadas medidas urgentes en el ámbito económico y para la protección de la salud pública. [acesso em 2020 maio 20]. Disponível em: https:// boe.es/boe/dias/2020/03/11/pdfs/BOE-A-2020-3434. pdf\#BOEn.

18. Ricart M. La Covid-19 monopoliza la sanidad privada en las áreas donde afecta más. [internet] La Vanguardia. 2020 abr 4. [acesso em 2020 maio 20]. Disponível em: https://www.lavanguardia.com/ vida/20200404/48287491681/covid-19-monopoliza-sanidad-privada-areas-afecta-mas.html.

19. Alsedo Q. 2.200 camas UCI estuvieron libres en España mientras 12.000 ancianos morían sin asistencia en las residências. [internet] El Mundo. 2020 abr 20 [acesso em 2020 maio 20]. Disponível em: https:// www.elmundo.es/ciencia-y-salud/salud/2020/04/ 19/5e9b579521efa084288b45de.html.

20. Rejo R. La sanidad privada calcula que tiene 2.200 camas de UCI sin usar en toda España. [internet] El Diario. 2020 abr 2 [acesso em 2020 maio 20]. Disponível em: https://www.eldiario.es/sociedad/sanidad-privada-calcula-UCI-Espana_0_1012449010.html.

21. Socorro EO. Sanidad pública y privada tendrán que reforzar su alianza en la era post Covid-19. [internet] El Espanol. 2020 abr 26 [acesso em 2020 maio 20]. https://www.elespanol.com/invertia/observatorios/ sanidad/20200426/sanidad-publica-privada-reforzar-alianza-post-covid-19/485202030_0.html.

22. Horgan-Jones J. Private hospitals in State to cater for Covid-19 cases only as pandemic spreads. [internet] The Irish Times. 2020 mar 25. [acesso em 2020 maio 20]. Disponível em: https://www.irishtimes. com/news/ireland/irish-news/private-hospitals-in-state-to-cater-for-covid-19-cases-only-as-pandemic-spreads-1.4211349.

23. Lehane M. Government seeks new deal with priva- te hospitals in pandemic. [internet] RTE. 2020 maio 29. [acesso em 2020 jul 30]. Disponível em: https:// www.rte.ie/news/coronavirus/2020/0529/1143415-coronavirus-private-hospitals/.

24. Kenny A. Arrangement with private hospitals met urgent objective, says Dept head. [internet] RTE. 2020 jul 2. [acesso em 2020 jul 30]. Disponível em: https:// www.rte.ie/news/ireland/2020/0602/1144866-dail-covid-19-committee/.

25. Itália. Decreto 400 de 23 agosto de 1998. Visto il decreto-legge 23 febbraio 2020, n. 6, recante «Misure urgenti in materia di contenimento e gestione dell'emergenza epidemiologica da COVID-19»e, in particolare, l'art. 3 [internet]. [acesso em 2020 maio 20]. Disponível em: https://www.gazzettaufficiale. it/eli/id/2020/03/09/20A01558/sg.

26. Ravennanotizie. Gli ospedali privati scendono in campo nell'emergenza Covid-19. Siglato accordo con la Regione: arrivano 3.750 posti letto in più. [internet] Ravenna Notizie. 2020 mar 21. [acesso em 2020 maio 20]. Disponível em: https://www.ravennanotizie.it/0-copertina/2020/03/21/gli-ospedali-privati-scendono-in-campo-nellemergenza-covid-19-siglato-accordo-con-la-regione-arrivano-3-750-posti-letto-in-piu/.

27. Telenord. Ospedali privati a supporto del Covid-19: già 40 pazienti dimessi da Villa Serena. [internet] Telenord. 2020 abr 27. [acesso em 2020 maio 20]. Disponível em: https://telenord.it/ospedali-privati-a-supporto-del-covid-19-gia-40-pazienti-dimessi-da-villa-serena.

28. Scagliarini M. Puglia, ospedali Covid, in campo i privati: altri 220 posti realizzati in tempo record. [internet] La Gazzetta del Mezzo Giorno. 2020 abr 2. [acesso em 2020 maio 20]. Disponível em: https://www. lagazzettadelmezzogiorno.it/news/home/1216234/ puglia-ospedali-covid-in-campo-i-privati-altri-220-posti-realizzati-in-tempo-record.html.

29. Abruzzoweb. Virus: in campo anche cliniche private, ospiteranno pazienti Covid e no Covid. [internet] Abruzzoweb. 2020 abr 9. [acesso em 2020 maio 20]. Disponível em: https://www.abruzzoweb.it/contenu- 
ti/coronavirus-coinvolte-cliniche-private-contratti-per-pazienti-covid-e-no-covid/727540-4/.

30. L'unione Sarda. Cliniche private Covid, Nieddu: "Scelta nell'interesse della salute dei sardi”. [internet] L'unione Sarda. 2020 abr 25. [acesso em 2020 maio 20]. Disponível em: https://www.unionesarda.it/articolo/news-sardegna/cagliari/2020/04/25/cliniche-private-covid-nieddu-scelta-nell-interesse-della-salute-136-1012237.html.

31. Chile. Ministério da Saúde. Unidad de Gestión Centralizada de Camas, UGCC. Período Enero 2014 - Diciembre 2017 [internet]. 2017 [acesso em 2020 maio 20]. Disponível em: https://www.minsal.cl/wp-content/uploads/2018/03/Informe-UGCC-2014-2018.pdf.

32. Chile. Ministério da Saúde. Dispone instrucciones para la coordinación de la red pública y privada de salud por parte de la subsecretaría de redes asistenciales. Santiago, 2020 abr 1. [acesso em 2020 maio 20]. Disponível em: https://www.minsal.cl/wp-content/ uploads/2020/04/1747890.pdf.

33. Chile. Ministério da Saúde. Dispone precios máximos que indica [internet]. 2020. [acesso em 2020 maio 20]. Disponível em: https://www.minsal.cl/wp-content/ uploads/2020/04/DO_1751692.pdf.

34. Claro H. El decreto que permite al Gobierno usar camas de hospitales privados por coronavirus. [internet] El Dinamo, 2020 mar 17. [acesso em 2020 maio 20]. Disponível em: https://www.eldinamo.com/nacional/2020/03/17/chile-coronavirus-alerta-sanitaria-uso-de-camas-privadas-sistema-publico/.

35. México. Secretaria de Saúde. Sector Salud suscribe convenio con hospitales privados para hacer frente a la pandemia de COVID-19. [acesso em 2020 maio 20]. Disponível em: https://www.gob.mx/salud/ prensa/105-sector-salud-suscribe-convenio-con-hospitales-privados-para-hacer-frente-a-la-pandemia-de-covid-19.

36. México. Todos juntos contra el COVID [internet]. [acesso em 2020 maio 20]. Disponível em: https:// coronavirus.gob.mx/todos-juntos/.
37. Machorro JC. Hospitales privados ponen $50 \%$ de sus camas por contingencia de COVID-19. [internet] Expok. 2020 abr 14. [acesso em 2020 maio 20]. Disponível em: https://www.expoknews.com/hospitales-privados-ponen-50-de-sus-camas-por-contingencia-de-covid-19/.

38. Peru. Decreto Legislativo ${ }^{\circ}{ }^{1466 .}$ Que, mediante Ley $n^{\circ} 31011$, el Congreso de la República ha delegado al Poder Ejecutivo la facultad de legislar en diversas materias para la atención de la emergencia sanitaria producida por el COVID - 19, por el término de cuarenta y cinco (45) días calendario [internet]. 2020. [acesso em 2020 maio 20]. Disponível em: https:// busquedas.elperuano.pe/normaslegales/decreto-legislativo-que-aprueba-disposiciones-para-fortalece-decreto-legislativo-n-1466-1865659-1/.

39. Vega ES. Clínicas privadas quedan bajo dirección del Minsa durante la emergência. [internet] OJO Publico. 2020 mar 17. [acesso em 2020 maio 20]. Disponível em: https://ojo-publico.com/1676/clinicas-privadas-quedan-bajo-direccion-del-minsa-durante-emergencia.

40. Gragnani J. Fila única por leitos de UTI? As propostas para fechar conta de desigualdade entre SUS e planos de saúde. [internet] BBC Brasil. 2020 abr 23. [acesso em 2020 maio 20]. Disponível em: https:// www.bbc.com/portuguese/brasil-52375980.

41. Agência Nacional de Saúde Suplementar. Processo $\mathrm{n}^{\circ}$ : $33910.010106 / 2020-60$ Voto $\mathrm{n}^{\circ} 11 / 2020 / D I-$ GES [internet]. 2020 [acesso em 2020 jul 20]. Disponivel em: https://static.congressoemfoco.uol.com. br/2020/06/76499__Voto-11.pdf.

42. Rio Grande do Sul. Secretaria de Estado da Saúde. Edital de Chamamento $n^{\circ}$ 015/2020 [internet]. 2020. [acesso em 2020 jul 30]. Disponível em: https://coronavirus-admin.rs.gov.br/upload/ arquivos/202008/17104642-edital-de-chamamento-n15.pdf.

43. Espírito Santo. Secretaria de Estado da Saúde. Portaria SESA no 77 de 30 de Abril de 2020. Definiu o princípio da complementariedade ao Sistema Único 
de Saúde (SUS), facultando ao Poder Público a possibilidade de recorrer aos serviços mantidos pela iniciativa privada, mediante contrato ou convênio, para garantir a cobertura assistencial à população nos casos em que a oferta pública for insuficiente. [internet]. 2020 [acesso em 2020 jul 30]. Disponível em: https://www.legisweb.com.br/legislacao/?id=394332.

44. Maranhão. Decreto n ${ }^{\circ} 35.797$ de 10 de maio de 2020. Determina, nos termos em que especifica, a requisição administrativa de 40 (quarenta) leitos das unidades hospitalares privadas localizadas, alternativamente, nos municípios de São Luís, São José de Ribamar, Paco do Lumiar e Raposa, e de 10 (dez) leitos de unidade de terapia intensiva (UTI) das unidades hospitalares privadas localizadas no município de Imperatriz. [acesso em 2020 jul 30]. Disponível em: https://www.corona.ma.gov.br/public/uploads/ arquivos/atos/33-5eb84b8d7c899.pdf.

45. Curitiba. Decreto $n^{\circ} 407$ de 13 de março de 2020. Dispõe sobre as condições e regras para a requisição de bens e serviços de assistência à saúde, visando o en- frentamento da emergência de saúde pública de importância nacional decorrente do Coronavírus no Município de Curitiba, para o atendimento integral aos usuários do SUS, mediante justa indenização à pessoas naturais e jurídicas [internet]. 2020 [acesso em 2020 jul 30]. Disponível em: https://www.legisweb. com.br/legislacao/?id=391117.

46. São Paulo. Decreto ${ }^{0} 59.396$ de 5 de Maio de 2020. Regulamenta a Lei ${ }^{\circ} 17.340$, de 30 de abril de 2020, que dispõe sobre medidas de proteção da saúde pública e de assistência social e outras medidas para o enfrentamento da Emergência de Saúde Pública em decorrência da Infecção Humana pelo Coronavírus (COVID-19) e determina outras providências [internet]. 2020 [acesso em 2020 jul 30]. Disponível em: http://diariooficial.imprensaoficial.com.br/nav_v5/ index.asp?c=1\&e=20200506\&p=1.

Recebido em 07/06/2020

Aprovado em 17/09/2020

Conflito de interesses: inexistente

Suporte financeiro: não houve 